Pacific Journal of Mathematics

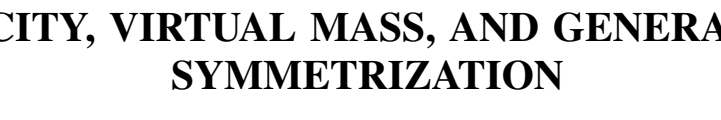




\section{CAPACITY, VIRTUAL MASS, AND GENERALIZED SYMMETRIZATION}

\section{E. PAYNe AND ALEXANDER Weinstein}

1. Introduction. A body of revolution $B$ can be symmetrized with respect to its axis of symmetry in a number of ways. One of these is the Schwarz symmetrization, which preserves the volume of $B$. Another is the Steiner symmetrization of the meridian section of $B$, which preserves the area of this section but in general decreases the volume. The influence of the Schwarz symmetrization on the capacity has been investigated by G. Pólya and G. Szegô,, [1]. More recently P. R. Garabedian and D. C. Spencer [2] discussed the same question for the virtual mass of bodies of revolution. In the present paper we shall study by a different and simpler method the behavior of the capacity and virtual mass under a more general type of symmetrization, which includes the Schwarz and Steiner symmetrizations as particular cases.

2. Definitions. Let the $(x, y)$-plane be the meridian plane of $B$, the $x$-axis being the axis of symmetry. The part of the meridian section of $B$ which lies in the upper half plane $y \geq 0$ is denoted by $D$. The complement of $D$ in the half plane is designated as $E$. We assume that $D$ is simply connected and that $E$ is a connected domain. The boundary of $D$ consists in general of a segment of the $x$-axis and a line $L$. We exclude the case where $L$ is a closed curve and lies entirely above the $x$-axis, as is the case in which $B$ is a torus. We assume $L$ to have at most a finite number of angular points.

We shall use in this paper some recent results of axially symmetric potential theory in $n$-dimensional space. This theory which is of mathematical interest in itself will be used here mainly as a tool to obtain results for bodies of revolution in three dimensions.

Let us henceforth consider our $(x, y)$-plane as the meridian plane of a body of revolution $B[n]$ in $n$-dimensions, $n=3,4,5, \ldots$. We assume that $B[n]$ has the same meridian section $D$ as our three-dimensional body $B=B$ [3]. All quantities considered hereafter are defined in the meridian plane and therefore are functions of $x$ and $y$ only. Actually we shall never use $B[n]$ but only its meridian section.

Received March 5, 1952. This research was conducted under the sponsorship of the Office of Naval Research.

Pacific J. Math. 2 (1952), 633-641 
Let $\phi(x, y)$ be an axially symmetric potential function defined for $y \geq 0$ and let $\psi(x, y)$ be the corresponding stream function. We have then the generalized Stokes-Beltrami equations

$$
y^{n-2} \frac{\partial \phi}{\partial x}=\frac{\partial \psi}{\partial y}, y^{n-2} \frac{\partial \phi}{\partial y}=-\frac{\partial \psi}{\partial x} .
$$

To emphasize the dependence of $\phi$ and $\psi$ on $n$ we shall often use the notations $\phi[n]$ and $\psi[n]$.

The volume $V[n]$ of $B[n]$ is given by

$$
V[n]=\omega_{n-1} \iint_{D} y^{n-2} d x d y
$$

where $\omega_{h}=2 \pi^{h / 2} / \Gamma(h / 2)$. We introduce the capacity $C[n]$ of $B[n]$ by the formula

$$
C[n]=\frac{\omega_{n-1}}{\omega_{n}} \iint_{E} y^{n-2}(\operatorname{grad} \phi[n])^{2} d x d y,
$$

where $\phi[n]$ is a potential which assumes the value unity on $L$ and vanishes as $\left(x^{2}+y^{2}\right)^{-(n-2) / 2}$ at infinity. It is obvious that (3) reduces to the classical definition of the capacity for $n=3$.

We define also the virtual mass $M[n]$ of $B[n]$ by the formula

$$
M[n]=\omega_{n-1} \iint_{E} y^{-(n-2)}(\operatorname{grad} \psi[n])^{2} d x d y
$$

The function $\psi[n]$ in (4) assumes the value $y^{n-1} /(n-1)$ on $L$ and vanishes at infinity like $y^{n-1}\left(x^{2}+y^{2}\right)^{-n / 2}$. Our definition of the virtual mass generalizes that of F. R. Garabedian and D. C. Spencer [2].

3. The correspondence principle and the fundamental formula. We use here a relationship due to A. Weinstein [4],

$$
\psi[n]=y^{n-1}(n-1)^{-1} \phi[n+2] .
$$

This equation shows that to each stream function $\psi[n]$ corresponds a welldefined potential $\phi[n+2]$. In particular to the stream function $\psi[n]$ in formula (4) corresponds a potential $\phi[n+2]$ which assumes the value unity on $L$ and vanishes as $\left(x^{2}+y^{2}\right)^{-n / 2}$ at infinity. In other words $\phi[n+2]$ is the electrostatic potential of $B[n+2]$. The substitution of (5) into (4) leads after an 
elementary integration by parts to the fundamental formula

$$
M[n]+V[n]=\pi^{n / 2}\left[(n-1) \Gamma\left(\frac{n}{2}+1\right)\right]^{-1} C[n+2],
$$

which we shall employ here in the study of the behavior of $M[n]$.

4. Generalized symmetrization. A line $x=$ constant, $y \geq 0$, intersects $L$ in $m$ points $y_{1}(x)>y_{2}(x)>y_{3}(x)>\cdots>y_{m}(x)>0$. The number of intersections $m$ usually depends on $x$. Let us consider the line $L_{q}$ defined by the equation

$$
y^{q}(x)=\sum_{k=1}^{m}(-1)^{k-1} y_{k}^{q}(x)
$$

where $q$ is a positive constant not necessarily an integer. The body of revolution $B_{q}[n]$ with section $D_{q}$ defined by its profile $L_{q}$ is said to be obtained by a symmetrization $S_{q}$. Let us note that $S_{n-1}$ can be considered as a Schwarz symmetrization of $B[n]$. On the other hand, under $S_{1}$ the meridian profile of $B[n]$ undergoes a Steiner symmetrization. Our main results are embodied in the following the orems:

I. $V[n]$ does not increase under $S_{q}$ for $0<q \leq n-1$ and does not decrease under $S_{q}$ for $q \geq n-1$. In particular, $V[n]$ remains invariant under $S_{n-1}$.

II. $C[n]$ does not increase under $S_{q}$ for $0<q \leq n-1$.

III. $M[n]$ does not increase under $S_{q}$ for $n-1 \leq q \leq n+1$.

Let us observe that by (6) Theorem III follows immediately from I and II. In order to prove Theorems I and II we shall first establish some useful inequalities.

5. Fundamental inequalities. Let $y_{1}>y_{2}>\cdots>y_{m}>0$ and let $q$ and $s$ be two positive numbers. We have then

$$
\left[\sum_{k=1}^{m}(-1)^{k-1} y_{k}^{q}\right]^{1 / q} \leq\left[\sum_{k=1}^{m}(-1)^{k-1} y_{k}^{q+s}\right]^{1 /(q+s)} \leq\left[\sum_{k=1}^{m} y_{k}^{q}\right]^{1 / q} .
$$

To prove the second inequality of (8) let us observe that it is sufficient to show that 


$$
\left[\sum_{k=1}^{m} y_{k}^{q+s}\right]^{1 /(q+s)} \leq\left[\sum_{k=1}^{m} y_{k}^{q}\right]^{1 / q} .
$$

Let us put $y_{k}^{q}=a_{k}$ and $(q+s) / q=r>1$. Then we need only show that

$$
a_{1}^{r}+a_{2}^{r}+\cdots+a_{m}^{r} \leq\left(a_{1}+a_{2}+\cdots+a_{m}\right)^{r}
$$

But this is a classical inequality [5, p. 32]. As to the first part " of (8) we give here a proof communicated to us by H. F. Weinberger [7]. This inequality does not seem to be mentioned in the available literature. Using again the notations in (10) and putting

$$
F\left(a_{1}, a_{2}, \cdots, a_{m}\right)=\sum_{k=1}^{m}(-1)^{k-1} a_{k}^{r}-\left[\sum_{k=1}^{m}(-1)^{k-1} a_{k}\right]^{r},
$$

we have to prove that, for $a_{1}>a_{2}>\cdots>a_{m}>0$ and $r>1$,

$$
F\left(a_{1}, a_{2}, \cdots, a_{m}\right) \geq 0
$$

This inequality is obviously true for $m=1$ and follows immediately if $m=2$ from inequality (10). Let us therefore assume that (12) holds if we replace $m$ by $m-2$; this is equivalent to assuming the inequality

$$
F\left(a_{2}, a_{2}, a_{3}, \cdots, a_{m}\right) \geq 0
$$

We have also

$$
F\left(a_{1}, a_{2}, \cdots, a_{m}\right)=F\left(a_{2}, a_{2}, a_{3}, \cdots, a_{m}\right)+\int_{a_{2}}^{a_{1}} \frac{\partial F}{\partial a_{1}} d a_{1}
$$

But from (11) we observe that

$$
\frac{1}{r} \frac{\partial F}{\partial a_{1}}=a_{1}^{r-1}-\left[\sum_{k=1}^{m}(-1)^{k-1} a_{k}\right]^{r-1}
$$

which shows that $\partial F / \partial a_{1}$ is nonnegative. Since the same holds by assumption for $F\left(a_{2}, a_{2}, a_{3}, \cdots, a_{m}\right)$ we obtain at once the required inequality (12).

${ }^{*}$ R. Bellman has pointed out that this inequality holds more generally with $y^{r}$ replaced by an arbitrary continuous convex function $f(y)$ defined for $y \geq 0$. 
6. The effect of the generalized symmetrization on $V[n]$. It follows immediately from (2) that

$$
V[n]=\omega_{n-1}(n-1)^{-1} \int_{\alpha}^{k}\left[\sum_{k=1}^{m}(-1)^{k-1} y_{k}^{n-1}\right] d x,
$$

where the integral is taken over the interval $(\alpha, \beta)$ bounded by the greatest and smallest values of $x$ on $L$. Let us apply the symmetrization $S_{q}$ defined by (7). The volume $V_{q}[n]$ is then given by

$$
V_{q}[n]=\omega_{n-1}(n-1)^{-1} \int_{\alpha}^{\beta}\left[\sum_{k=1}^{m}(-1)^{k-1} y_{k}^{q}\right]^{n-1 / q} d x .
$$

By (8) we see that for $q \leq n-1$ we have

$$
V_{q}[n] \leq \omega_{n-1}(n-1)^{-1} \int_{\alpha}^{\beta}\left[\sum_{k=1}^{m}(-1)^{k-1} y_{k}^{n-1}\right] d x=V[n] .
$$

On the other hand for $q \geq n-1$ we have again by (8)

$$
V_{q}[n] \geq \omega_{n-1}(n-1)^{-1} \int_{\alpha}^{\beta}\left[\sum_{k=1}^{m}(-1)^{k-1} y_{k}^{n-1}\right] d x=V[n] .
$$

The formulas (18) and (19) establish the proof of Theorem 1 of $\$ 4$.

7. The effect of generalized symmetrization on $C[n]$. In studying the behavior of $C[n]$ under the symmetrization $S_{q}$ we shall generalize to a certain extent the procedure given by Pólya and Szegö for the Steiner symmetrization $[1, \mathrm{p} .182]$. Let us introduce a Cartesian system $(x, y, z)$ and consider a surface $z(x, y)$ defined in a large half circle $A$ enclosing $D$. We assume $z(x, y)$ to be a function positive throughout $A$ and vanishing on the circular portion of its boundary. The particular function $z$ which we shall consider will assume a constant positive value $z_{0}$ in the subdomain $D$ of $A$. This value will be the maximum of $z(x, y)$ in $A$. We further assume that $z(x, y)$ is analytic outside $D$. The surface $z=z(x, y)$ except for its flat portion may also be defined as a surface $y=y(x, z)$ in a certain domain $G$ of the $(x, z)$-plane. However, $y(x, z)$ may not be a single-valued function of $x$ and $z$. For this reason we must consider as in [1] the surfaces $y_{k}(x, z)(k=1,2, \cdots, m)$, where

$$
y_{1}(x, z)>y_{2}(x, z)>\cdots>y_{m}(x, z)>0 \text {. }
$$


These surfaces taken together with the flat portion constitute the surface $z(x, y)$.

Let us consider the integral

$$
I=\iint_{G} \sum_{k=1}^{m} y_{k}^{n-2}(x, z)\left[1+\left(\frac{\partial y_{k}}{\partial x}\right)^{2}+\left(\frac{\partial y_{k}}{\partial z}\right)^{2}\right]^{1 / 2} d x d z .
$$

Let us first apply the symmetrization $S_{n-1}$ by putting

$$
y_{*}^{n-1}=\sum_{k=1}^{m}(-1)^{k-1} y_{k}^{n-1},
$$

and consider the integral

$$
l_{*}=\iint_{G} y_{*}^{n-2}\left[1+\left(\frac{\partial y_{*}}{\partial x}\right)^{2}+\left(\frac{\partial y_{*}}{\partial z}\right)^{2}\right]^{1 / 2} d x d z .
$$

We prove now that

$$
I \geq I_{*}
$$

In fact by substituting (21) into (22) and computing $\partial y_{*} / \partial x$ and $\partial y_{*} / \partial z$ we obtain the formula

$$
\begin{array}{r}
I_{*}=\iint_{G}\left\{\left[\sum_{k=1}^{m}(-1)^{k-1} y_{k}^{n-1}\right]^{2(n-2) /(n-1)}+\left[\sum_{k=1}^{m}(-1)^{k-1} y_{k}^{n-2} \frac{\partial y_{k}}{\partial x}\right]^{2}\right. \\
+\left[\sum_{k=1}^{m}(-1)^{k-1} y_{k}^{n-2} \frac{\partial y_{k}}{\partial z}\right]^{2} d x d z .
\end{array}
$$

According to the inequality (8), $I_{*}$ will not diminish if we replace the first square bracket in (24) by $\left[\sum_{k=1}^{m} y_{k}^{n-2}\right]^{2}$. Upon applying the Minkowski inequality we find that the integrand in $I_{*}$ is not greater than the integrand in $l$; this proves formula (23).

Let us observe that

$$
I=\iint_{A} y^{n-2}\left[1+z_{x}^{2}+z_{y}^{2}\right] 1 / 2 d x d y-\iint_{D} y^{n-2} d x d y
$$


the last integral being the contribution from the flat part of the $z$ surface. We now insert into (25) the expression $z(x, y)=\epsilon \Phi(x, y), \epsilon$ being a small positive number and $\Phi$ satisfying the same conditions as $z$. This substitution yields

$$
I=\iint_{A-D} y^{n-2} d x d y+\left(\epsilon^{2} / 2\right) \iint_{A} y^{n-2}\left(\Phi_{x}^{2}+\Phi_{y}^{2}\right) d x d y+O\left(\epsilon^{4}\right) .
$$

According to inequality (23), $I$ does not increase under $S_{n-1}$. The first integral in (26) is obviously equal to the same integral taken over the symmetrized domain $A_{*}-D_{*}$, where $A_{*}=A$. By letting $\epsilon$ tend to zero we conclude in the usual way [1] that the integral

$$
\iint_{A} y^{n-2}\left(\Phi_{x}^{2}+\Phi_{y}^{2}\right) d x d y
$$

does not increase under $S_{n-1}$. If we let the radius of the half circle bounding $A$ tend to infinity we obtain the same statement for a function $\Phi$ which vanishes at infinity, providing that the integral converges. In particular if we take for $\Phi$ a function which is equal to unity in $D$ and equal to the electrostatic potential $\phi[n]$ in $E$ we find that $C[n]$ does not increase under $S_{n-1}$.

In order to prove that $C[n]$ does not increase under $S_{q}$ for $0<q \leq n-1$ let us observe that under $S_{q}$ the line $L$ bounding $D[n]$ goes into a line $L_{q}$ which has by the inequalities (8) the following property: if $q_{1}<q_{2}$ then the domain $D_{q_{1}}[n]$ bounded by $L_{q_{1}}$ has no points outside the domain $D_{q_{2}}[n]$ bounded by $L_{q_{2}}$. We denote the capacities corresponding to these domains by $C_{q_{1}}[n]$ and $C_{q_{2}}[n]$, respectively. It is a well known property of the ordinary three-dimensional capacity that if one body contains another body the former has the larger capacity. The proof of this statement is based essentially on the variational definition of the capacity. The same property holds obviously for all values of $n$. We therefore have $C_{q_{1}}[n] \leq C_{q_{2}}[n]$. In particular $C_{q}[n] \leq C_{n-1}[n]$. As we have already proved $C_{n-1}[n] \leq C[n]$ we obtain the result

$$
C_{q}[n] \leq C[n],
$$$$
0<q \leq n-1,
$$

which concludes the proof of Theorem II of $\$ 4$. As already mentioned in 34 , Theorem III follows immediately as a corollary of I and II.

8. Stainer's Symmetrization of the meridian section with respect to the $y$-axis. We shall consider briefly a symmetrization of the domain $D$ with respect to the $y$-axis defined by the classical equation 


$$
2 x=\sum_{k=1}^{m}(-1)^{k-1} x_{k}
$$

In a manner similar to that used in 8 we find that $V[n]$ remains invariant and $C[n]$ and $M[n]$ do not increase under such a symmetrization.

9. Concluding remarks. All results of $\$ 4$ can be extended to the case of two dimensional bodies which are symmetric with respect to the $x$-axis. It should be noted that these results hold for $C[2]$ as long as the radius of $A$ remains finite. It has already been proven $[1,2]$ that $C[2]$ and $M[2]$ do not increase under $S_{1}$ and also that $C[3]$ and $M[3]$ do not increase under $S_{2}$. These cases are included in our Theorems II and III. We note also that formula (6) appears in an equivalent form for $n=2$ and $n=3$ in papers by G. I. Taylor [6] and M. Schiffer and G. Szego" [3], where $C[4]$ and $C[5]$ are (up to a constant factor) called dipole coefficients. No attempt was made in these papers to study the behavior of the dipole coefficients under symmetrization. However, it was recognized in [3] that they are increasing set functions, a fact which becomes almost obvious in our theory of generalized electrostatics (see $\$ 7$ ). Finally let us remark that in $\$ 2$ we have introduced the $(x, y)$-plane as the meridian plane of an $n$-dimensional space. But since all quantities are defined in terms of $x$ and $y$, the index $n$ appearing in our formulas need not be restricted to integral values . In fact it can easily be seen that all our formulas and results remain valid for all real positive values of $n$ greater than two. For such values of $n$ our results are mathematical statements about certain integrals such as $V[n], C[n]$, and $M[n]$ which are associated with the generalized Stokes-Beltrami equations.

\section{REFERENCES}

1. G. Pólya and G. Szegő, Isoperimetric inequalities in mathematical physics, Annals of Mathematics Studies No. 27, Princeton University Press, 1951.

2. P. R. Garabedian and D. C. Spencer, Extremal methods in cavitational flow, Technical Report No. 3, Applied Mathematics and Statistics Laboratory, Stanford University, 1951.

3. M. Schiffer and G. Szegó, Virtual mass and polarization, Trans. Amer. Math. Soc. 67 (1949), 130 - 205.

4. A. Weinstein, On cracks and dislocations in shafts under torsion, Quart. Appl. Math. 10 (1952), 77-81; The method of singularities in the physical and in the hodograph plane, Fourth Symp. Appl. Math. (in print). 
5. G. H. Hardy, J. E. Littlewood and G. Pólya, Inequalities, Cambridge, England, 1934.

6. G. I. Taylor, The energy of a body moving in an infinite fluid with an application to airships, Proc. Roy. Soc. London Ser. A, 120 (1928), 21-33.

7. H. Weinberger, An inequality with alterating signs, Proc. Nat. Acad. Sci., U.S.A. 38 (1952), 611 - 613.

Institute for Fluid Dynamics and Applied Mathematics

UNIVERSITY OF MARYLAND 



\section{PACIFIC JOURNAL OF MATHEMATICS}

\section{EDITORS}

\section{R. M. RoBINSON \\ University of California Berkeley 4, California}

\author{
*R. P. Dilmorth \\ Califomia Institute of Technology \\ Pasadena 4, California
}

E. F. BeckenbaCh, Managing Editor

University of California

Los Angeles 24, California

${ }^{*}$ During the absence of Herbert Busemann in 1952.

\section{ASSOCIATE EDITORS}

$\begin{array}{llll}\text { R. P. DILWORTH } & \text { P. R. HALMOS } & \text { BØRGE JESSEN } & \text { J. J. STOKER } \\ \text { HERBERT FEDERER } & \text { HEINZ HOPF } & \text { PAUL LÉVY } & \text { E. G. STRAUS } \\ \text { MARSHALL HALL } & \text { R. D. JAMES } & \text { GEORGE PÓLYA } & \text { KÖSAKU YOSIDA }\end{array}$

\section{SPONSORS}

UNIVERSITY OF BRITISH COLUMBIA

CALIFORNIA INSTITUTE OF TECHNOLOGY

UNIVERSITY OF CALIFORNIA, BERKELEY

UNIVERSITY OF CALIFORNIA, DAVIS

UNIVERSITY OF CALIFORNIA, LOS ANGELES

UNIVERSITY OF CALIFORNIA, SANTA BARBARA

OREGON STATE COLLEGE

UNIVERSITY OF OREGON

\author{
UNIVERSITY OF SOUTHERN CALIFORNIA \\ STANFORD UNIVERSITY \\ WASHINGTON STATE COLLEGE \\ UNIVERSITY OF WASHINGTON \\ AMERICAN MATHEMATICAL SOCIETY \\ NATIONAL BUREAU OF STANDARDS, \\ INSTITUTE FOR NUMERICAL ANALYSIS
}

Mathematical papers intended for publication in the Pacific Journal of Miathematics should be typewritten (double spaced), and the author should keep a complete copy. Manuscripts may be sent to any of the editors. All other communications to the editors should be addressed to the managing editor, E. F. Beckenbach, at the address given above.

Authors are entitled to receive 100 free reprints of their published papers and may obtain additional copies at cost.

The Pacific Journal of Mathematics is published quarterly, in March, June, September, and December, by the University of California, Berkeley 4, California. The price per volume (4 numbers) is \$8.00; single issues, $\$ 2.50$. Special price to individual faculty members of supporting institutions and to individual members of the American Mathematical Society: $\$ 4.00$ per volume; single issues, $\$ 1.25$.

Subscriptions, orders for back numbers, and changes of address should be sent to the publishers, University of California Press, Berkeley 4, California.

Printed at Ann Arbor, Michigan. Entered as second class matter at the Post Office, Berkeley, California.

\section{UNIVERSITY OF CALIFORNIA PRESS • BERKELEY AND LOS ANGELES}




\section{Pacific Journal of Mathematics}

\section{Vol. 2, No. $4 \quad$ April, 1952}

Shmuel Agmon, On the singularities of Taylor series with reciprocal coefficients .................................... 431

Richard Arens, A generalization of normed rings ............... 455

Iacopo Barsotti, Intersection theory for cycles of an algebraic variety . . . . 473

Leonard M. Blumenthal, Two existence theorems for systems of linear inequalities ..................................... 523

Frank Herbert Brownell, III, Translation invariant measure over separable Hilbert space and other translation spaces................. 531

J. W. S. Cassels, On a paper of Niven and Zuckerman ............... 555

Nelson Dunford, Spectral theory. II. Resolutions of the identity .......... 559

Eugene Lukacs and Otto Szász, On analytic characteristic functions ...... 615

W. A. Mersman, Evaluation of an integral occurring in servomechanism theory.......................................... 627

Lawrence Edward Payne and Alexander Weinstein, Capacity, virtual mass, and generalized symmetrization......................... 633

Choy-Tak Taam, The boundedness of the solutions of a differential equation in the complex domain ...................................... 643 